\title{
ON SEMI B-FREDHOLM OPERATORS
}

\author{
M. BERKANI* \\ Université Mohammed I, Faculté des Sciences, Département de Mathématiques, Oujda, Maroc \\ e-mail:berkani@sciences.univ-oujda.ac.ma \\ and M. SARIH \\ Université Ibn Tofail, Faculté des Sciences, Département de Mathématiques, Kénitra, Maroc
}

(Received 8 February, 2000)

\begin{abstract}
An operator $T$ on a Banach space is called 'semi B-Fredholm' if for some $n \in \mathbf{N}$ the range $R\left(T^{n}\right)$ of $T^{n}$ is closed and the induced operator $T_{n}$ on $R\left(T^{n}\right)$ semi-Fredholm. Semi B-Fredholm operators are stable under finite rank perturbation, and subject to the spectral mapping theorem; on Hilbert spaces they decompose as sums of nilpotent and semi-Fredholm operators. In addition some recent generalizations of the punctured neighborhood theorem turn out to be consequences of Grabiner's theory of 'topological uniform descent'.
\end{abstract}

1991 Mathematics Subject Classification. 47A53, 47A55.

1. Introduction. The first author [1] has studied B-Fredholm operators on Banach spaces, defined as operators for which some power $T^{n}$ has closed range $R\left(T^{n}\right)$, on which the restriction $T_{n}$ is Fredholm, in the sense of having null space $N(T)$ of finite dimension $\alpha(T)$ and range $R(T)$ of finite codimension $\beta(T)$; the difference $\operatorname{ind}(T)=\alpha(T)-\beta(T)$ is known as the index. In this note we extend our study to "semi B-Fredholm" operators, for which $T_{n}$ is either upper or lower semiFredholm, in the sense that either $N(T)$ is finite dimensional and $R(T)$ closed, or $R(T)$ is closed of finite codimension. We shall see that the semi B-Fredholm operators $\operatorname{SBF}(X)$ on a Banach space $X$ in general properly contain the semi-Fredholm operators $S F(X)$ and we prove that each semi B-Fredholm operator is a quasiFredholm operator in the sense defined by Mbekhta and Muller in [10], a definition which coincides with the definition given in the case of operators acting on a Hilbert space by Labrousse in [9]. Conversely a quasi-Fredholm operator for which there exists an integer $n$ such that $N(T) \cap R\left(T^{n}\right)$ is of finite dimension or $N\left(T^{n}\right)+R(T)$ is of finite codimension is a semi B-Fredholm operator.

In Theorem 2.6 and in the case of operators acting on a Hilbert space $H$ we prove that $T \in L(H)$ is a semi B-Fredholm operator if and only if $T=Q \oplus F$, where $Q$ is a nilpotent operator and $F$ is a semi-Fredholm operator. But we do not know if this characterization is still valid in the case of operators acting on a Banach space. In Proposition 2.7, we prove that if $T \in L(X)$ is a semi B-Fredholm operator and if $F \in L(X)$ is a finite dimensional operator then $T+F$ is also a semi B-Fredholm operator.

*Supported by a grant of CGRI-Belgium. 
In the third section we consider some recent generalizations of the "punctured neighborhood theorem" obtained by Schmoeger [12], Harte [4] and Harte and Lee [5], and we show that those generalizations are a particular case of a result of Grabiner on operators of topological uniform descent [3, Theorem 4.7]. For definitions and properties of operators of topological uniform descent we refer the reader to the paper of Grabiner [3].

In the fourth section we prove a spectral mapping theorem for semi B-Fredholm operators, more precisely in Theorem 4.5, for $T \in L(X)$ and $f$ an analytic function on the usual spectrum $\sigma(T)$ of $T$, which is non-constant on any connected component of $\sigma(T)$, we prove that $f\left(\sigma_{S B F}(T)\right)=\sigma_{S B F}(f(T))$, where $\sigma_{S B F}(T)=\{\lambda \in \mathbf{C} / T-$ $\lambda I \notin S B F(X)\}$.

In the sequel if $E$ and $F$ are two vector spaces, the notation $E \simeq F$ will mean that $E$ and $F$ are isomorphic. If $E, F$ are vector subspaces of the same vector space $H$ we shall write $E={ }_{e} F$ if there exist two finite dimensional vector subspaces $G_{1}, G_{2}$ of $H$ such that $E \subset F+G_{1}$ and $F \subset E+G_{2}$.

\section{Properties of semi B-Fredholm operators}

Proposition 2.1. Let $T \in L(X)$. If there exists an integer $n \in \mathbf{N}$ such that $R\left(T^{n}\right)$ is closed and such that the operator $T_{n}$ is an upper semi-Fredholm (resp. a lower semiFredholm) operator, then $R\left(T^{m}\right)$ is closed, $T_{m}$ is an upper semi-Fredholm (resp. a lower semi-Fredholm) operator, for each $m \geq n$. Moreover, if $T_{n}$ is a Fredholm operator, then $T_{m}$ is a Fredholm operator and ind $\left(T_{m}\right)=\operatorname{ind}\left(T_{n}\right)$ for each $m \geq n$.

Proof. As $T_{n}: R\left(T^{n}\right) \rightarrow R\left(T^{n}\right)$ is a semi-Fredholm operator, then for each $m \geq n$ the operator $T_{n}^{m-n}: R\left(T^{n}\right) \rightarrow R\left(T^{n}\right)$ is also a semi-Fredholm operator. Hence $R\left(T_{n}^{m-n}\right)=R\left(T^{m}\right)$ is closed in $R\left(T^{n}\right)$. Since $R\left(T^{n}\right)$ is also closed in $X$, then $R\left(T^{m}\right)$ is closed in $X$. If $T_{n}$ is an upper semi-Fredholm operator then $\alpha\left(T_{n}\right)<\infty$. Since $N\left(T_{m}\right)=N(T) \cap R\left(T^{m}\right) \subset N(T) \cap R\left(T^{n}\right)=N\left(T_{n}\right)$, we have $\alpha\left(T_{m}\right)<\infty$. Since $R\left(T^{m+1}\right)$ is closed, $T_{m}$ is an upper semi-Fredholm operator. In the same way, if $T_{n}$ is a lower semi-Fredholm operator, then $\beta\left(T_{n}\right)<\infty$. As $R\left(T_{n}\right)=R\left(T^{n+1}\right)$, there exists a finite dimensional subspace $F$ of $R\left(T^{n}\right)$ such that $R\left(T^{n}\right)=F+R\left(T^{n+1}\right)$. Then $R\left(T^{m}\right)=T^{m-n}(F)+R\left(T^{m+1}\right)$ and $R\left(T_{m}\right)=R\left(T^{m+1}\right)$ is of finite codimension in $R\left(T^{m}\right)$. Consequently $\beta\left(T_{m}\right)<\infty$ and $T_{m}$ is a lower semi-Fredholm operator. Moreover if $T_{n}$ is a Fredholm operator, then $T_{m}$ is a Fredholm operator. From [6, Lemma 3.5] we have: $\frac{N(T) \cap R\left(T^{n}\right)}{N(T) \cap R\left(T^{n+1}\right)} \simeq \frac{N\left(T^{n+1}\right)+R(T)}{N\left(T^{n}\right)+R(T)}$ and from [6, Lemma 3.2] we have $\frac{R\left(T^{n}\right)}{R\left(T^{n+1}\right)} \simeq \frac{X}{R(T)+N\left(T^{n}\right)} \quad$ and $\quad \frac{R\left(T^{n+1}\right)}{R\left(T^{n+2}\right)} \simeq \frac{X}{R(T)+N\left(T^{n+1}\right)} . \quad$ Hence $\quad \alpha\left(T_{n}\right)-\alpha\left(T_{n+1}\right)=\beta\left(T_{n}\right)-$ $\beta\left(T_{n+1}\right)$ and so $\operatorname{ind}\left(T_{n+1}\right)=\operatorname{ind}\left(T_{n}\right)$. It follows then that $\operatorname{ind}\left(T_{m}\right)=\operatorname{ind}\left(T_{n}\right)$ for each $m \geq n$.

Definition 2.2. Let $T \in L(X)$. If for some integer $n, R\left(T^{n}\right)$ is closed and the operator $T_{n}$ is an upper semi-Fredholm (resp. a lower semi-Fredholm) operator, then $T$ is called an upper semi B-Fredholm (resp. a lower semi B-Fredholm) operator. A semi B-Fredholm operator is an upper or a lower semi B-Fredholm operator.

From the definition of semi B-Fredholm operators, it is easily seen that all nilpotent operators and all bounded linear projections are semi B-Fredholm operators. Hence the class $\operatorname{SBF}(X)$ of semi B-Fredholm operators contains the class of semi- 
Fredholm operators as a proper subclass. Note also that obviously every B-Fredholm operator is a semi B-Fredholm operator.

REMARK. Let $T \in L(X)$ be a semi B-Fredholm operator and $n$ any integer such that $R\left(T^{n}\right)$ is closed and $T_{n}$ is a semi-Fredholm operator. Then we can define the index ind $(T)$ of $T$ as the index of the semi-Fredholm operator $T_{n}$. From Proposition 2.1 , this definition is independent of the choice of the integer $n$. Furthermore if $T$ is a Fredholm operator this reduces to the usual definition of the index.

Definition 2.3. [9] Let $T \in L(X)$ and let

$$
\Delta(T)=\left\{n \in \mathbf{N}: \quad \forall m \in \mathbf{N}, \quad m \geq n \Rightarrow\left(R\left(T^{n}\right) \cap N(T)\right) \subset\left(R\left(T^{m}\right) \cap N(T)\right)\right\} .
$$

Then the degree of stable iteration $\operatorname{dis}(T)$ of $T$ is defined as $\operatorname{dis}(T)=\inf \Delta(T)$ (with $\operatorname{dis}(T)=\infty$ if $\Delta(T)=\emptyset)$.

Definition 2.4. Let $T \in L(X)$. Then $T$ is called a quasi-Fredholm operator of degree $d$ if there is an integer $d \in \mathbf{N}$ such that

(a) $\operatorname{dis}(T)=d$,

(b) $R\left(T^{n}\right)$ is a closed subspace of $X$ for each integer $n \geq d$,

(c) $R(T)+N\left(T^{d}\right)$ is a closed subspace of $X$.

In the sequel $Q F(d)$ will denote the set of quasi-Fredholm operators of degree $d$.

REMARK. It is easily seen that this definition is equivalent to the definitions given by Mbekhta and Muller in [10, p. 143], Poon in [11] and in the case of Hilbert spaces it is equivalent to the definition given by Labrousse in [9, Definition 3.1.2]. From [3, Theorem 3.2] we see also that a quasi-Fredholm operator of degree $d$ is an operator of topological uniform descent for $n \geq d$.

Proposition 2.5. Let $T \in L(X)$. Then $T$ is an upper semi B-Fredholm (resp. a lower semi B-Fredholm) operator if and only if there exists an integer $d \in \mathbf{N}$ such that $T \in Q F(d)$ and such that $N(T) \cap R\left(T^{d}\right)$ is of finite dimension (resp. $R(T)+N\left(T^{d}\right)$ ) is of finite codimension.

Proof. Suppose that $T$ is a semi B-Fredholm operator, and let $n \in \mathbf{N}$ be such that $R\left(T^{n}\right)$ is closed and the operator $T_{n}$ is a semi-Fredholm operator. If $T_{n}$ is an upper semi-Fredholm operator, then

$$
\alpha\left(T_{n}\right)=\operatorname{dim} N\left(T_{n}\right)=\operatorname{dim}\left(N(T) \cap R\left(T^{n}\right)\right)<\infty .
$$

If $m \geq n$, then $N(T) \cap R\left(T^{m}\right) \subset N(T) \cap R\left(T^{n}\right)$. Since $\operatorname{dim}\left(N(T) \cap R\left(T^{n}\right)\right)<\infty$, the sequence $\left(N(T) \cap R\left(T^{p}\right)\right)_{p}$ is a stationary sequence for $p$ large enough. This shows that $d=\operatorname{dis}(T) \in \mathbf{N}$, and $\operatorname{dim}\left(N(T) \cap R\left(T^{d}\right)\right)<\infty$. As $R\left(T^{m}\right)$ is closed for each integer $m \geq n$, using [10, Lemma 12] we deduce that $R\left(T^{m}\right)$ is closed, for each integer $m \geq d$. Moreover we have $R(T)+N\left(T^{d}\right)=\left(T^{d}\right)^{-1}\left(R\left(T^{d+1}\right)\right)$ is a closed subspace of $X$. Consequently $T \in Q F(d)$ with $\operatorname{dim}\left(N(T) \cap R\left(T^{d}\right)\right)<\infty$.

In the same way if $T_{n}$ is a lower semi-Fredholm operator, then $\beta\left(T_{n}\right)=$ $\operatorname{dim} \frac{X}{R(T)+N\left(T^{n}\right)}<\infty$. Hence the sequence $\left(R(T)+N\left(T^{p}\right)\right)_{p}$ is a stationary sequence for $p$ large enough. Since 


$$
\frac{N(T) \cap R\left(T^{p}\right)}{N(T) \cap R\left(T^{p+1}\right)} \simeq \frac{N\left(T^{p+1}\right)+R(T)}{N\left(T^{p}\right)+R(T)},
$$

$d=\operatorname{dis}(T) \in \mathbf{N}$ and $R(T)+N\left(T^{d}\right)$ is of finite codimension. Moreover Proposition 2.1 shows that $R\left(T^{m}\right)$ is closed, for each integer $m \geq n$. By [10, Lemma 12], $R\left(T^{m}\right)$ is closed for each integer $m \geq d$. Hence $T \in Q F(d)$.

Conversely, since $T \in Q F(d), R\left(T^{n}\right)$ is closed for each $n \geq d$. Moreover, as $\alpha\left(T_{d}\right)=\operatorname{dim}\left(N(T) \cap R\left(T^{d}\right)\right)$ and $\beta\left(T_{d}\right)=\operatorname{dim} \frac{X}{R(T)+N\left(T^{d}\right)}$, if $\operatorname{dim}\left(N(T) \cap R\left(T^{d}\right)\right)<\infty$ (resp. $\left.\operatorname{codim}\left(R(T)+N\left(T^{d}\right)\right)<\infty\right)$, the operator $T_{d}$ is an upper semi-Fredholm (resp. a lower semi-Fredholm) operator. Hence $T_{d}$ is a semi-Fredholm operator and $T$ is a semi B-Fredholm operator.

As each semi B-Fredholm operator acting on a Hilbert space is a quasi-Fredholm operator in the sense of Labrousse [9], using the Kato decomposition defined in [9], we give now a fundamental characterization of semi B-Fredholm operators in the case of Hilbert spaces.

Theorem 2.6. Let $H$ be a Hilbert space and let $T \in L(H)$. Then $T$ is a semi $B$ Fredholm operator if and only if there exist two closed subspaces $M$ and $N$ of $H$ such that $H=M \oplus N$ and

(i) $T(N) \subset N$ and $T_{\mid N}$ is a nilpotent operator,

(ii) $T(M) \subset M$ and $\left(T_{\mid M}\right)$ is a semi-Fredholm operator.

Proof. Suppose that $T$ is a semi B-Fredholm operator. From Proposition 2.5 it is a quasi-Fredholm operator. Hence from [9, Théoréme 3.2.1] there exists two closed subspaces $M, N$ of $H$ and an integer $d \in \mathbf{N}$ such that $H=M \oplus N$ and

(i) $T(N) \subset N$ and $T_{\mid N}$ is a nilpotent operator of degree $d$,

(ii) $T(M) \subset M, R\left(T_{\mid M}\right)$ is closed and $N\left(T_{\mid M}\right) \subset \cap_{m} R\left(\left(T_{\mid M}\right)^{m}\right)$.

Moreover from formula (3.2.23) in [9, Théoréme 3.2.1] we have that $N\left(T_{\mid M}\right)=$ $N(T) \cap R\left(T^{d}\right)$. We have also $R\left(T_{\mid M}\right) \oplus N=R(T)+N\left(T^{d}\right)$. If $T$ is an upper semi BFredholm operator, then $N\left(T_{\mid M}\right)=N(T) \cap R\left(T^{d}\right)$ is of finite dimension. Since $R\left(T_{\mid M}\right)$ is closed $T_{\mid M}$ is an upper semi-Fredholm operator.

If $T$ is a lower semi B-Fredholm operator, then there exists a finite dimensional subspace $F$ of $H$ such that $H=F \oplus R\left(T_{\mid M}\right) \oplus N$. Let $P_{M}: H \rightarrow M$ be the linear projection along $N$ onto $M$. Then $M=P_{M}(F)+R\left(T_{\mid M}\right)$. This shows that $R\left(T_{\mid M}\right)$ is of finite codimension in $M$. Hence $R\left(T_{\mid M}\right)$ is closed and $T_{\mid M}$ is a lower semi-Fredholm operator.

Conversely suppose that $H=M \oplus N$ with the conditions (i) and (ii) satisfied. Since $T_{\mid M}$ is a semi-Fredholm operator, then $R\left(T^{d}\right)=R\left(\left(T_{\mid M}\right)^{d}\right)$ is closed in $M$, and so it is also closed in $H$ and $T_{d}=\left(T_{\mid M}\right)_{d}$. Using Propsition 2.1 and the fact that $T_{\mid M}$ is a semi-Fredholm operator we see that $T_{d}$ is a semi-Fredholm operator and so $T$ is a B-Fredholm operator.

Setting $T_{\mid N}=Q, T_{\mid M}=F$, we see that $T=Q \oplus F$, where $Q$ is a nilpotent operator and $F$ is a semi-Fredholm operator.

Proposition 2.7. Let $T \in L(X)$ be a semi B-Fredholm operator and let $F \in L(X)$ be a finite dimensional operator. Then $T+F$ is also a semi B-Fredholm operator.

Proof. We know from [8] that $T+F$ is a quasi-Fredholm operator. Let $n \in \mathbf{N}$ be such that $n \geq \max (\operatorname{dis} T$, $\operatorname{dis}(T+F))$. If $T$ is an upper semi B-Fredholm operator, 
then we have $N(T) \cap R\left(T^{n}\right)$ is of finite dimension. As $\left(N(T+F) \cap R\left((T+F)^{n}\right)\right)={ }_{e}$ $N(T) \cap R\left(T^{n}\right)$, it follows that $N(T+F) \cap R\left((T+F)^{n}\right)$ is of finite dimension. Hence $T+F$ is a semi B-Fredholm operator.

If $T$ is a lower semi B-Fredholm operator, then $\frac{R\left(T^{n}\right)}{R\left(T^{n+1}\right)}$ is of finite dimension. As $\left.R\left((T+F)^{n}\right)\right)={ }_{e} R\left(T^{n}\right)$ and $\left.R\left((T+F)^{n+1}\right)\right)={ }_{e} R\left(T^{n+1}\right)$, we see that $\frac{R\left((T+F)^{n}\right)}{R\left((T+F)^{n+1}\right)}$ is of finite dimension. Hence $T+F$ is a semi B-Fredholm operator.

REMARK. We mention that a result of Poon [[11], Theorem 10] shows that for a semi B-Fredholm operator $T$, $\lim _{n \rightarrow \infty} \gamma\left(T^{n}\right)^{1 / n}$ exists, where $\gamma(T)$ is the reduced minimum modulus of $T$, and this limit is equal to the stability radius of $T$. For more details see [11].

3. On Grabiner's punctured neighborhood theorem. In [3] Grabiner has defined and studied a class of linear operators acting on a Banach space called operators of topological uniform descent. Among other things Grabiner has proved the following theorem [3, Theorem 4.7].

Theorem 3.1. Suppose that $T$ is a bounded operator with topological uniform descent for $n \geq d$ on the Banach space $X$, where $n, d \in \mathbf{N}$, and $V$ is a bounded operator that commutes with $T$. If $V-T$ is sufficiently small and invertible, then

(i) $V$ has closed range and uniform descent for $p \geq 0$,

(ii) $\operatorname{dim} \frac{N\left(V^{p+1}\right)}{N\left(V^{p}\right)}=\operatorname{dim} \frac{N\left(T^{d+1}\right)}{N\left(T^{d}\right)}$, for each integer $p \geq 0$,

(iii) $\operatorname{dim} \frac{R\left(V^{p}\right)}{R\left(V^{p+1}\right)}=\operatorname{dim} \frac{R\left(T^{d}\right)}{R\left(T^{d+1}\right)}$, for each integer $p \geq 0$.

From this theorem we obtain easily the following Corollary.

Corollary 3.2. Suppose that $T$ is a bounded operator with topological uniform descent for $n \geq d$ on the Banach space $X$, and that $V$ is a bounded operator that commutes with T. If $V-T$ is sufficiently small and invertible, then the following hold.

(i) If $N(T) \cap R\left(T^{d}\right)$ is of finite dimension, then $V$ is an upper semi-Fredholm operator and $\alpha(V)=\operatorname{dim}\left(\left(N(T) \cap R\left(T^{d}\right)\right)\right.$.

(ii) If $R(T)+N\left(T^{d}\right)$ is of finite codimension, then $V$ is a lower semi-Fredholm operator and $\beta(V)=\operatorname{dim} \frac{X}{R(T)+N\left(T^{d}\right)}$.

Proof. As $T$ is an operator of topological uniform descent, $R(V)$ is closed by Theorem 3.1. Since $\operatorname{dim} \frac{N\left(T^{d+1}\right)}{N\left(T^{d}\right)}=\operatorname{dim}\left(N(T) \cap R\left(T^{d}\right)\right)$ and $\operatorname{dim} \frac{R\left(T^{n}\right)}{R\left(T^{n+1}\right)}=\operatorname{dim} \frac{X}{R(T)+N\left(T^{d}\right)}$, then from Theorem 3.1 we have $\alpha(V)=\operatorname{dim}\left(\left(N(T) \cap R\left(T^{d}\right)\right), \beta(V)=\operatorname{dim} \frac{X}{R(T)+N\left(T^{d}\right)}\right.$.

This proves the Corollary.

As a semi B-Fredholm operator is an operator of topological uniform descent, Corollary 3.1 gives a punctured neighborhood theorem for semi B-Fredholm operators. We see also that Corollary 3.1 generalizes the punctured neighborhood theorem stated in the case of semi-Fredholm operators in [7, Theorem 3 and Theorem 5].

Proposition 3.3. Let $T \in L(X)$.

(i) If there exists an integer $n \in \mathbf{N}$ such that $R\left(T^{n}\right) \cap N(T)$ is of finite dimension and $R\left(T^{n}\right)+N(T)$ is closed, then $T$ is an upper semi B-Fredholm operator. 
(ii) If there exist an integer $n \in \mathbf{N}$ such that $R\left(T^{n}\right)+N(T)$ is of finite codimension, then $T$ is an operator of topological uniform descent.

(iii) If there exist an integer $n \in N$ such that $R\left(T^{n}\right)+N(T)$ is closed and $R\left(T^{n}\right) \cap N(T)=R\left(T^{m}\right) \cap N(T)$, for all $m \geq n$, then $T$ is an operator of topological uniform descent.

Proof. (i) Suppose that there is an integer $n \in \mathbf{N}$ such that $R\left(T^{n}\right) \cap N(T)$ is of finite dimension and $R\left(T^{n}\right)+N(T)$ is closed. To prove that $T$ is an upper semi BFredholm operator, it is enough to prove that $R\left(T^{n}\right)$ and $R\left(T^{n+1}\right)$ are closed. As $R\left(T^{n}\right) \cap N(T)$ is of finite dimension, there exist a finite codimensional closed subspace $E$ of $N(T)$ such that $N(T)=R\left(T^{n}\right) \cap N(T) \oplus E$. We know that $R\left(T^{n}\right)$, equipped with the operator range topology, is a Banach space continuously imbedded in $X$. Consider the map $S: R\left(T^{n}\right) \times E \rightarrow R\left(T^{n}\right)+N(T)$, defined by $S((x, y))=x+y$. Since $R\left(T^{n}\right) \cap E=R\left(T^{n}\right) \cap N(T) \cap E=\{0\}, S$ is injective. As $S$ is continuous and onto, from the open mapping theorem it is bicontinuous. Hence $S\left(R\left(T^{n}\right) \times\{0\}\right)=$ $R\left(T^{n}\right)$ is closed in $R\left(T^{n}\right)+N(T)$, and so $R\left(T^{n}\right)$ is closed in $X$. Moreover as $R(T)+$ $N\left(T^{n}\right)=\left(T^{(n-1)}\right)^{-1}\left(R\left(T^{n}\right)+N(T)\right)$, we see that $R(T)+N\left(T^{n}\right)$ is a closed subspace of $X$. Since $R\left(T^{n}\right)$ is also a closed subspace of $X$, by [7, Lemma 331] it follows that $R\left(T^{n+1}\right)$ is a closed subspace of $X$.

(ii) If $m \geq n$, then $R\left(T^{n}\right)+N(T) \subset R(T)+N\left(T^{n}\right) \subset R(T)+N\left(T^{m}\right)$. Hence the sequence $\left(R(T)+N\left(T^{m}\right)\right)_{m}$ is a stationary sequence for $m$ large enough. Hence $T$ is an operator of uniform descent. Moreover if $m \geq n$, then from [6, Lemma 3.2] we have $\frac{R\left(T^{m}\right)}{R\left(T^{m+1}\right)} \simeq \frac{X}{R(T)+N\left(T^{m}\right)}$ so that $R\left(T^{m+1}\right)$ is of finite codimension in $R\left(T^{m}\right)$. Hence $R\left(T^{m+1}\right)$ is closed in $R\left(T^{m}\right)$ when this last space is equipped with the operator range topology. By [3, Theorem 3.2], $T$ is an operator of topological uniform descent.

(iii) Since $R\left(T^{m}\right) \cap N(T)=R\left(T^{n}\right) \cap N(T)$, for all $m \geq n$, T is an operator of uniform descent for $m \geq n$. Moreover as $R(T)+N\left(T^{n}\right)=\left(T^{(n-1)}\right)^{-1}\left(R\left(T^{n}\right)+N(T)\right)$, it follow that $R(T)+N\left(T^{n}\right)$ is a closed subspace of $X$. By [3, Theorem 3.2], it follows that $T$ is an operator of topological uniform descent for $m \geq n$.

We show now that the generalizations of the punctured neighborhood theorem obtained by Schmoeger [12, Theorem 1], Harte [4, Theorem 5], Harte and Lee [5, Theorem 4] are all consequences of Grabiner's theorem. We show also that Grabiner's theorem can be applied to answer a question of Harte and Lee [5, p. 273].

(a) In [12, Theorem 1] Schmoeger considers an operator $T$ in $L(X)$ such that $R\left(T^{2}\right)$ is closed and $R(T) \cap N(T)$ is of finite dimension. Hence $R(T)+N(T)=$ $(T)^{-1}\left(R\left(T^{2}\right)\right)$ is a closed subspace of $X$. By part (i) of Proposition 3.3, $T$ is an upper semi B-Fredholm operator.

(b) In [4, Theorem 5], Harte and in [5, Theorem 4], Harte and Lee consider an operator $T$ in $L(X)$ satisfying condition (i) or conditions (i) and (ii) of Proposition 3.2. In the two cases the operator $T$ is an operator of topological uniform descent.

We see then that in all those cases, the operator under consideration is an operator of topological uniform descent. By Corollary 3.2 we obtain the desired result.

(c) In [5, p. 273] Harte and Lee consider an operator $T$ satisfying the conditions of part (iii) in Proposition 3.3 and they ask what happens under these hypothesis. In this case $T$ is also an operator of topological uniform descent and Corollary 3.2 gives an answer to their question. 
4. A spectral mapping theorem for semi B-Fredholm operators. Let $T \in S B F(X)$ and let $\rho_{S B F}(T)=\{\lambda \in \mathbf{C} /(T-\lambda I) \in S B F(X)\}$ be the semi B-Fredholm resolvent of $T$ and let $\sigma_{S B F}(T)=\{\lambda \in \mathbf{C} / T-\lambda I \notin S B F(X)\}$ be the semi B-Fredholm spectrum of $T$.

Proposition 4.1. Let $T \in L(X)$. The B-Fredholm spectrum $\sigma_{S B F}(T)$ of $T$ is a closed subset of $\mathbf{C}$ contained in the usual spectrum $\sigma(T)$ of $T$.

Proof. If $\lambda \notin \sigma(T)$, then $(T-\lambda I)$ is invertible and $\lambda \notin \sigma_{S B F}(T)$. Hence $\sigma_{S B F}(T) \subset \sigma(T)$. If $\alpha \notin \sigma_{S B F}(T)$, then $S=T-\alpha I$ is a semi B-Fredholm operator. If $\lambda$ is small and $\lambda$ is not equal to zero, by [10, Table 2, p. 144] $S-\lambda I$ is a quasi-Fredholm operator. Moreover from Theorem 3.1 we have that

$$
\begin{aligned}
\operatorname{dim}\left(N(S-\lambda I) \cap R\left((S-\lambda I)^{n}\right)\right) & =\operatorname{dim} \frac{N\left((S-\lambda I)^{n+1}\right)}{N\left((S-\lambda I)^{n}\right)}=\operatorname{dim} \frac{N\left(S^{n+1}\right)}{N\left(S^{n}\right)} \\
& =\operatorname{dim}\left(N(S) \cap R\left(S^{n}\right)\right), \\
\operatorname{dim} \frac{X}{N\left((S-\lambda I)^{n}\right)+R(S-\lambda I)} & =\operatorname{dim} \frac{R\left((S-\lambda I)^{n}\right)}{R\left((S-\lambda I)^{n+1}\right)}=\operatorname{dim} \frac{R\left(S^{n}\right)}{R\left(S^{n+1}\right)} \\
& =\operatorname{dim} \frac{X}{N\left(S^{n}\right)+R(S)},
\end{aligned}
$$

and so $S-\lambda I$ is a semi B-Fredholm operator. Hence $\rho_{S B F}(T)$ is open in $\mathbf{C}$ or, equivalently, $\sigma_{S B F}(T)$ is closed.

Proposition 4.2. Let $T \in L(X)$. The following properties are equivalent.

(i) $T$ is a semi B-Fredholm operator.

(ii) $T^{m}$ is a semi B-Fredholm operator, for each integer $m>0$.

(iii) $T^{m}$ is a semi B-Fredholm operator, for some integer $m>0$.

Proof. (i) $\Rightarrow$ (ii). Suppose that $T$ is a semi B-Fredholm operator, and let $d=\operatorname{dis}(T)$, then Proposition 2.1 shows that $R\left(T^{m d}\right)$ is a closed subspace of $X$ and the operator $T_{m d}$ is a semi-Fredholm operator. Hence $\left(T^{m}\right)_{d}=\left(T_{m d}\right)^{m}$ is also a semiFredholm operator, so that $T$ is a semi B-Fredholm operator.

(ii) $\Rightarrow$ (iii). This is obvious.

(iii) $\Rightarrow$ (i). Suppose that $T^{m}$ is a semi B-Fredholm operator, for some integer $m>0$. Hence there exists an integer $n$ such that $R\left(T^{n m}\right)$ is closed and the operator $\left(T^{m}\right)_{n}: R\left(T^{n m}\right) \rightarrow R\left(T^{n m}\right)$ is a semi-Fredholm operator. Since $\left(T_{m n}\right)^{m}=\left(T^{m}\right)_{n}$, we see that $\left(T_{m n}\right)^{m}$ is a semi-Fredholm operator. Hence $T_{m n}$ is also a semi-Fredholm operator and $T$ is a semi B-Fredholm operator.

Proposition 4.3. Let $S, T, A, B \in L(X)$ be mutually commuting operators, satisfying $T A+B S=I$. Then $T S \in S B F(X)$ if and only if $T, S \in \operatorname{SBF}(X)$.

Proof. Let $n \in \mathbf{N}$. From [10, Lemma 1] $R\left((T S)^{n}\right)$ is closed if and only if $R\left(T^{n}\right)$ and $R\left(S^{n}\right)$ are closed. Moreover from [10, Lemmma 8] we have the following result.

$$
R\left((T S)^{n}\right) \cap N(T S)=R\left(T^{n}\right) \cap N(T)+R\left(S^{n}\right) \cap N(S) .
$$


Hence $R\left((T S)^{n}\right) \cap N(T S)$ is of finite dimension if and only if $R\left(T^{n}\right) \cap N(T)$ and $R\left(S^{n}\right) \cap N(S)$ are of finite dimension. We have also from [10, Lemma 4] that

$$
\sup \left(\operatorname{dim} \frac{R\left(T^{n}\right)}{R\left(T^{n+1}\right)}, \operatorname{dim} \frac{R\left(S^{n}\right)}{R\left(S^{n+1}\right)}\right) \leq \operatorname{dim} \frac{R\left((T S)^{n}\right)}{R\left((T S)^{n+1}\right)} \leq \operatorname{dim} \frac{R\left(T^{n}\right)}{R\left(T^{n+1}\right)}+\operatorname{dim} \frac{R\left(S^{n}\right)}{R\left(S^{n+1}\right)}
$$

Hence $T S$ is a semi B-Fredholm operator if and only if $T$ and $S$ are semi B-Fredholm operators.

From this proposition we have immediately the following result.

Corollary 4.4. Let $P(X)=\left(X-\lambda_{1} I\right)^{m_{1}} \ldots\left(X-\lambda_{n} I\right)^{m_{n}}$ be a polynomial with complex coefficients. Then $P(T)=\left(T-\lambda_{1} I\right)^{m_{1}} \ldots\left(T-\lambda_{n} I\right)^{m_{n}}$ is a semi B-Fredholm operator if and only if $\forall i, 1 \leq i \leq n,\left(T-\lambda_{i} I\right)$ is a semi B-Fredholm operator.

THEOREM 4.5. Let $T \in L(X)$ and $f$ be an analytic function in a neighborhood of the usual spectrum $\sigma(T)$ of $T$, that is non-constant on any connected component of $\sigma(T)$. Then $f\left(\sigma_{S B F}(T)\right)=\sigma_{S B F}(f(T))$.

Proof. Let $\mu \in \sigma_{S B F}(T)$. Since $\sigma(T)$ is a compact subset of $\mathbf{C}$, the function $f(z)-f(\mu)$ possesses at most a finite number of zeros in $\sigma(T)$. Hence

$$
f(z)-f(\mu)=(z-\mu)^{m_{0}}\left(z-\lambda_{1}\right)^{m_{1}} \ldots\left(z-\lambda_{n}\right)^{m_{n}} g(z),
$$

where $g(z)$ is a non-vanishing analytic function on $\sigma(T)$. Using the functional calculus (cf. [2]) we have

$$
f(T)-f(\mu) I=(T-\mu I)^{m_{0}}\left(T-\lambda_{1} I\right)^{m_{1}} \ldots\left(T-\lambda_{n} I\right)^{m_{n}} g(T),
$$

where $g(T)$ is an invertible operator. Hence $(g(T))^{-1}$ is a semi B-Fredholm operator. If $f(T)-f(\mu) I$ is a semi B-Fredholm operator, by Proposition 4.3 applied to $f(T)-f(\mu) I$ and $(g(T))^{-1}$, we have that $(T-\mu I)^{m_{0}}\left(T-\lambda_{1} I\right)^{m_{1}} \ldots\left(T-\lambda_{n} I\right)^{m_{n}}=$ $(f(T)-f(\mu) I)(g(T))^{-1}$ is a semi B-Fredholm operator. By Corollary $4.4, T-\mu I$ is a semi B-Fredholm operator, a fact which contradicts our assumption. Hence $f(\mu) \in \sigma_{S B F}(f(T))$ and $f\left(\sigma_{S B F}(T)\right) \subset \sigma_{S B F}(f(T))$.

Conversely let $\alpha \in \sigma_{S B F}(f(T))$, so that $\alpha \in \sigma(f(T))$. Hence there exists $\mu \in \sigma(T)$ such that $f(\mu)=\alpha$. We have $f(z)-f(\mu)=(z-\mu)^{m_{0}}\left(z-\mu_{1}\right)^{m_{1}} \ldots\left(z-\mu_{n}\right)^{m_{n}} g(z)$, where $\mathrm{g}$ is a non-vanishing function analytic on $\sigma(T)$. Hence

$$
f(T)-f(\mu) I=(T-\mu I)^{m_{0}}\left(T-\mu_{1} I\right)^{m_{1}} \ldots\left(T-\mu_{n} I\right)^{m_{n}} g(T)=f(T)-\alpha I .
$$

As $f(T)-\alpha I$ is not a semi B-Fredholm operator, by Corollary 4.4 there exists $\beta \in\left\{\mu, \mu_{1}, \ldots, \mu_{n}\right\}$ such that $T-\beta I$ is not a semi B-Fredholm operator. Hence $f(\beta)=\alpha$ with $\beta \in \sigma_{S B F}(T)$, so that $f\left(\sigma_{S B F}(T)\right)=\sigma_{S B F}(f(T))$. This proves the theorem.

Acknowledgements. The authors would like to thank the referee for his useful comments and observations. 


\section{REFERENCES}

1. M. Berkani, On a class of quasi-Fredholm operators, Integral Equations Operator Theory 34 (1999), 244-249.

2. N. Dunford and J. T. Schwartz, Linear operators: Part 1 (Interscience, New York, 1971).

3. S. Grabiner, Uniform ascent and descent of bounded operators, J. Math. Soc. Japan 34 No. 2 (1982), 317-337.

4. R. Harte, On Kato non-singularity, Studia Math. 117 No. 2 (1996), 107-114.

5. R. Harte and W. Y. Lee, A note on the punctured neighborhood theorem, Glasgow Math. J. 39 (1997), 269-273.

6. M. A. Kaashoek, Ascent, descent, nullity and defect; a note on a paper by A. E. Taylor, Math. Ann. 172 (1967), 105-115.

7. T. Kato, Perturbation theory for nullity, deficiency and other quantities of linear operators. J. Anal. Math. 6 (1958), 261-322.

8. J. J. Koliha, M. Mbekhta, V. Muller and P. W. Poon, Corrigendum and addendum: On the axiomatic theory of the spectrum II, Studia Math. 130 No. 2 (1998), 193-198.

9. J. P. Labrousse, Les opérateurs quasi-Fredholm: une généralisation des opérateurs semi-Fredholm, Rend. Circ. Mat. Palermo (2) 29 (1980), 161-258.

10. M. Mbekhta and V. Muller, On the axiomatic theory of the spectrum II, Studia Math. 119 No. 2 (1996), 129-147.

11. P. W. Poon, On the stability radius of a quasi-Fredholm operator, Proc. Amer. Math. Soc., 126 No. 4 (1998) 1071-1080.

12. C. Schmoeger, On a generalized punctured neighborhood theorem in $L(X)$, Proc. Amer. Math. Soc. 123 No. 4 (1995), 1237-1240. 